\title{
Cutaneous soft tissue tumors: diagnostically disorienting epithelioid tumors that are not epithelial, and other perplexing mesenchymal lesions
}

\author{
Cody S. Carter ${ }^{1} \cdot$ Rajiv M. Patel $\mathbb{1}^{2}$
}

Received: 12 August 2019 / Revised: 8 October 2019 / Accepted: 8 October 2019 / Published online: 4 November 2019

(c) The Author(s), under exclusive licence to United States \& Canadian Academy of Pathology 2019

\begin{abstract}
Cutaneous soft tissue tumors with epithelioid features present a diagnostic challenge given that many entities in this category are rare, and they show morphologic overlap with significantly more common cutaneous epithelial and melanocytic neoplasms. The challenge is compounded by overlapping expression of epithelial or melanocytic markers in some of these entities. A broad spectrum of primary cutaneous epithelioid soft tissue tumors exists, including benign and malignant counterparts of tumors with various differentiation including melanocytic, peripheral nerve sheath, angiomatous, fibrohistiocytic, and myoid or myoepithelial, in addition to translocation-associated tumors lacking a derivative tissue type. Given this spectrum, an initial targeted immunohistochemical panel for epithelioid dermal and subcutaneous neoplasms is recommended, covering a broad spectrum of differentiation. In diagnostically challenging cases, select molecular studies can be employed to make critical distinctions between entities sharing morphologic and immunohistochemical properties. Due to sometimes marked differences in prognosis and treatment, knowledge and familiarity with epithelioid soft tissue tumors is key for any surgical pathologist who evaluates skin and subcutaneous biopsies and excision specimens. This concise review provides brief descriptions, key diagnostic features, and important modern ancillary studies for the diagnosis of non-epithelial, non-melanocytic cutaneous tumors that can exhibit a prominent degree of epithelioid morphology.
\end{abstract}

\section{Introduction}

Epithelioid soft tissue tumors are so named because of their resemblance to epithelial cells, composed of round, ovoid, or polygonal cells containing abundant cytoplasm with either central or eccentric rounded nuclei. They can pose a challenge to many dermatopathologists and surgical pathologists due to their overlapping features with more common primary cutaneous epithelial neoplasms (carcinomas and adnexal tumors, among others) and both benign and malignant melanocytic lesions. Furthermore, many of these tumors are rarely encountered in daily practice compared to epithelial and melanocytic neoplasia. Because of their

Rajiv M. Patel

rajivpat@med.umich.edu

1 Department of Pathology and Human Anatomy, Loma Linda University, Loma Linda, CA, USA

2 Department of Pathology and Dermatology, Michigan Medicine, University of Michigan, Ann Arbor, MI, USA overlapping morphologic features, immunohistochemistry and molecular studies play an important role in elucidating the type of differentiation of a particular epithelioid soft tissue lesion. Morphologic variants add an additional layer of complexity, as many dermal soft tissue tumors have a variety of morphologic variants based on cytoplasmic quality (i.e. clear cell variants), or stroma (i.e. myxoid and sclerosing/hyalinized variants). As such, an initial focused immunohistochemical screening panel for epithelioid dermal and subcutaneous neoplasms is recommended for tumors that lack a distinct morphology, which includes a broadspectrum cytokeratin such as MNF116 or combination of cytokeratins AE1/AE3 and CAM5.2, as well as CD34, S100, or SOX10, smooth muscle actin (SMA), and CD45 (Table 1). Endothelial markers CD31 or ERG can also be considered in cases with prominent atypia, and EMA can also be useful as a second-line marker in some of the entities to be discussed. Conceptually, cellular differentiation provides a useful schema to organize one's understanding and knowledge base on this topic, with further subdivision into benign and malignant categories within each line of differentiation, and this review is thus organized in this manner. 
Table 1 Recommended immunohistochemistry screening panel for challenging cutaneous epithelioid tumors

\begin{tabular}{|c|c|c|c|c|c|c|}
\hline & Cytokeratins & $\begin{array}{l}\text { S100 } \\
\text { or SOX10 }\end{array}$ & SMA & CD45 & CD34 & CD31 or ERG \\
\hline Carcinoma & + & - & - & - & - & - \\
\hline Melanoma & - & + & - & - & - & - \\
\hline Lymphoma & - & - & - & + & - & - \\
\hline $\begin{array}{l}\text { Epithelioid fibrous and } \\
\text { fibrohistiocytic tumors }\end{array}$ & - & - & - & - & - & - \\
\hline $\begin{array}{l}\text { Epithelioid peripheral nerve } \\
\text { sheath tumors }\end{array}$ & - & + & - & - & - & - \\
\hline $\begin{array}{l}\text { Epithelioid tumors with myoid or } \\
\text { myoepithelial differentiation }\end{array}$ & $+1-$ & $+1-$ & $+1-$ & - & - & - \\
\hline Epithelioid vascular tumors & - & - & + & - & + & + \\
\hline
\end{tabular}

$+:$ positive staining; $-:$ negative staining; $+/-$ : positive staining in subset
A number of the soft tissue tumors described herein are owing to the seminal works of Dr. Franz Enzinger, who was either the principle or senior author on many of the initial descriptions of these entities, many of which were described decades before readily accessible molecular genetic testing that led to discovery of their recurrent genetic alterations. This practical, concise review is meant to highlight some of the recent genetic developments in these tumors, as well as the clinical, prognostic, morphologic, and immunophenotypic features of the tumors. In addition, important diagnostic pitfalls and differential diagnoses will be highlighted, as appropriate.

\section{Epithelioid fibrous and fibrohistiocytic tumors}

\section{Epithelioid fibrous histiocytoma}

Though previously described as a morphologic variant of benign fibrous histiocytoma, with recent advances in molecular genetics, epithelioid fibrous histiocytoma (EFH) is now considered a distinct pathologic entity based on its recurrent $A L K$ gene alterations $[1,2]$. These tumors most commonly arise in the dermis of the extremities of young to middle-aged adults, presenting as an erythematous papule. They follow a benign clinical course and do not recur after complete excision.

Epithelioid fibrous histiocytomas consist of a dermal proliferation of epithelioid, stellate, spindled, and plasmacytoid cells with moderately abundant eosinophilic cytoplasm which stands out against a pale eosinophilic, often hyalinized collagenous stroma (Fig. 1a-c). The cells contain monotonous ovoid or reniform nuclei with vesicular chromatin and small, pinpoint nucleoli. Binucleate or trinucleate cells are not uncommon, which show similar morphology to the mononuclear cells, differing from foreign body-type multinucleated giant cells. The nodules are typically well circumscribed and lack peripheral collagen trapping commonly seen in benign fibrous histiocytomas. In many cases, EFH exhibits an epidermal collarette at the edges of the lesion (Fig. 1a, b). Dilated, branching thin-walled vascular channels are common, and perivascular cell whorling is variably prominent.

As previously stated, $A L K$ gene rearrangements were discovered in the vast majority of these tumors, corresponding with diffuse, strong cytoplasmic ALK expression by immunohistochemistry (Fig. 1d) [1, 2]. Several fusion partners have subsequently been described, most commonly SQSTM1 and VCL [3]. Interrogation of the $A L K$ gene has led to an expansion of the morphologic spectrum of EFH to include 2 cases demonstrating a densely cellular, fascicular spindle cell morphology, but these features did not correlate with a common fusion partner [4]. Also of note, EFH are commonly positive for EMA but typically negative for both high and low-molecular weight cytokeratins, S100, and CD34 (Table 2). They are also commonly positive for TFE3 , but next-generation sequencing (NGS) panels have failed to detect any TFE3 gene alterations, and therefore the expression pattern has been postulated to be the result of upstream activation of the TFE3 gene [4].

The differential diagnosis includes cutaneous syncytial myoepithelioma (CSM), which closely mimics EFH in morphology and exhibits an overlapping immunophenotype. CSM shares positivity for EMA, but can be differentiated by its positivity for cytokeratins and S100, and lack of ALK positivity. In cases showing equivocal immunohistochemistry results, rearrangements in EWSRI by fluorescence in situ hybridization (FISH) can be demonstrated in most cases of CSM, or $A L K$ rearrangements can be identified in EFH. Other considerations in the differential diagnosis include conventional dermatofibroma (benign fibrous histiocytoma), which shows peripheral collagen trapping and is negative for EMA and ALK, as well as cellular 
Fig. 1 Epithelioid fibrohistiocytic tumors. a-d Epithelioid fibrous histiocytoma. At low magnification, epithelioid fibrous histiocytomas $(\mathrm{EFH})$ are well circumscribed dermal papules lacking peripheral collagen trapping, and often exhibit an epidermal collarette (a, b). Upon closer inspection, EFH consists of a dermal proliferation of epithelioid and plasmacytoid cells with moderately abundant eosinophilic cytoplasm, reniform nuclei, and hyalinized collagenous stroma (c). The epithelioid cells show diffuse cytoplasmic positivity for ALK (d). e-g Cellular neurothekeoma. Cellular neurothekeomas consist of a mixed population of spindled and epithelioid cells with abundant amphophilic cytoplasm arranged in cords and nests, separated by thick collagen bundles (e). The tumors cells in cellular neurothekeoma are positive for $\mathrm{NKI} / \mathrm{C} 3$ (f) and PGP9.5 (g). Epithelioid fibrous papules are benign dome-shaped lesions typically arising on the face, consisting of plump epithelioid stromal cells with abundant eosinophilic or clear cytoplasm within the superficial dermis $(\mathbf{h})$
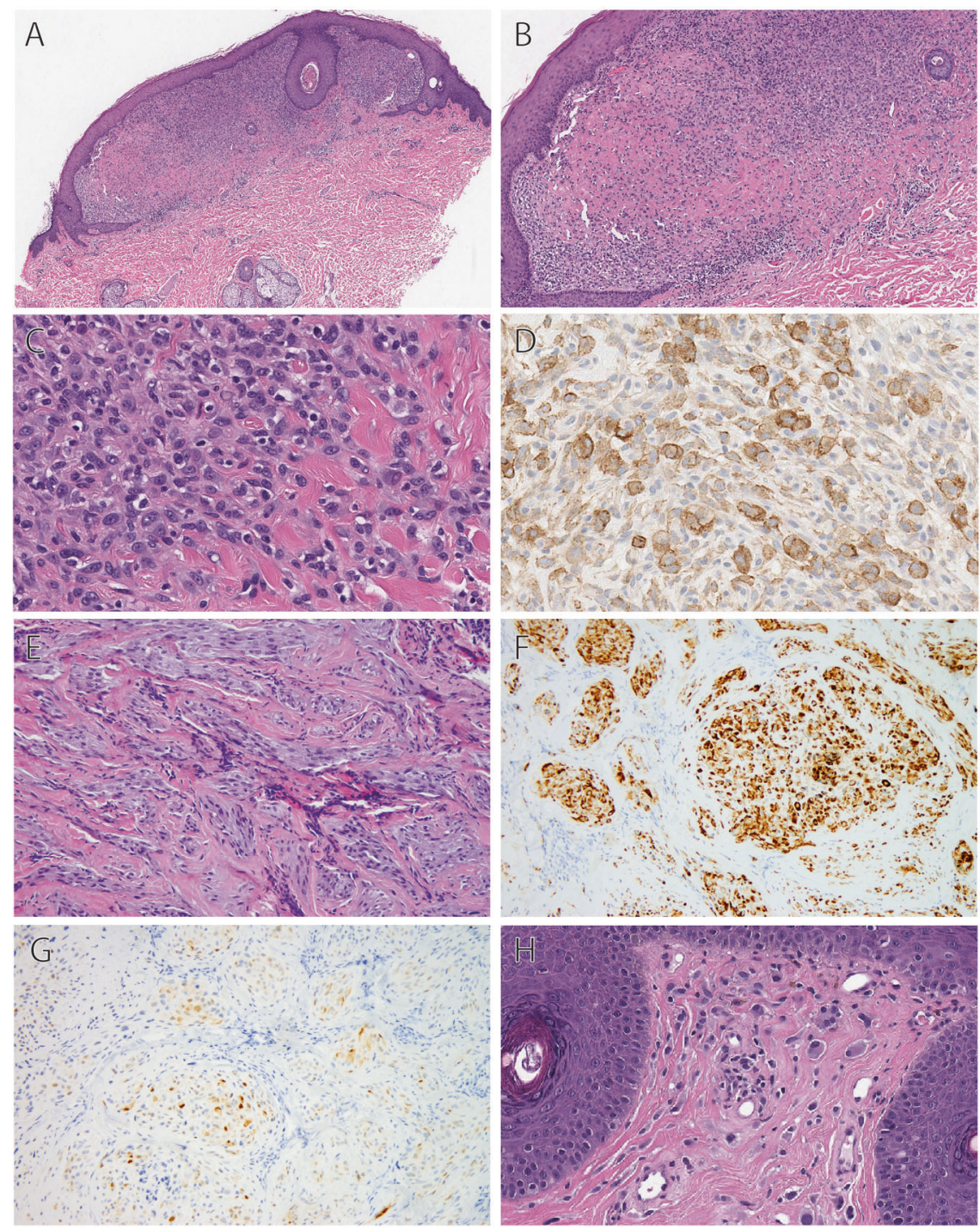

neurothekeoma, which typically shows a more nested pattern and is positive for PGP9.5 and NKI/C3, while negative for EMA and ALK. Melanocytic lesions, particularly Spitz nevi, also enter the differential diagnosis, but can be differentiated by their positivity for melanocytic markers $\mathrm{S} 100$ protein, SOX10, HMB45, and Melan-A. It is important to note that a small minority of Spitz nevi are positive for ALK by immunohistochemistry, which correlate with $A L K$ fusions detectable by FISH [5].

\section{Cellular neurothekeoma}

Cellular neurothekeoma is a benign cutaneous soft tissue tumor which most often involves the head and neck or extremities of young adults with a slight female predominance [6]. They only rarely recur, usually in the case of incomplete excision. Though the name of this entity implies nerve sheath differentiation, this is now considered a misnomer as they do not show phenotypic or ultrastructural evidence of nerve sheath cells, and instead share genetic overlap with benign fibrous histiocytomas and other fibrohistiocytic lesions [7].

Histologically, cellular neurothekeomas are predominantly dermal tumors, and they often consist of a mixed population of spindled and epithelioid cells with abundant amphophilic cytoplasm, but can occasionally be composed of a marked predominance of epithelioid cells, particularly in the desmoplastic variant [8]. A large subset of lesions can show superficial subcutaneous extension. The constituent cells are arranged in nests and cords separated by thick collagen bundles, and they contain ovoid nuclei with fine chromatin, small nucleoli, and abundant eosinophilic or amphophilic cytoplasm (Fig. 1e). The aforementioned desmoplastic variant has a denser collagenous stroma, which separates the cells into smaller nests and single cells. The myxoid variant does not enter the differential diagnosis, as the constituent cells of that variant are more uniformly spindled. Cytologic atypia, osteoclast-like 


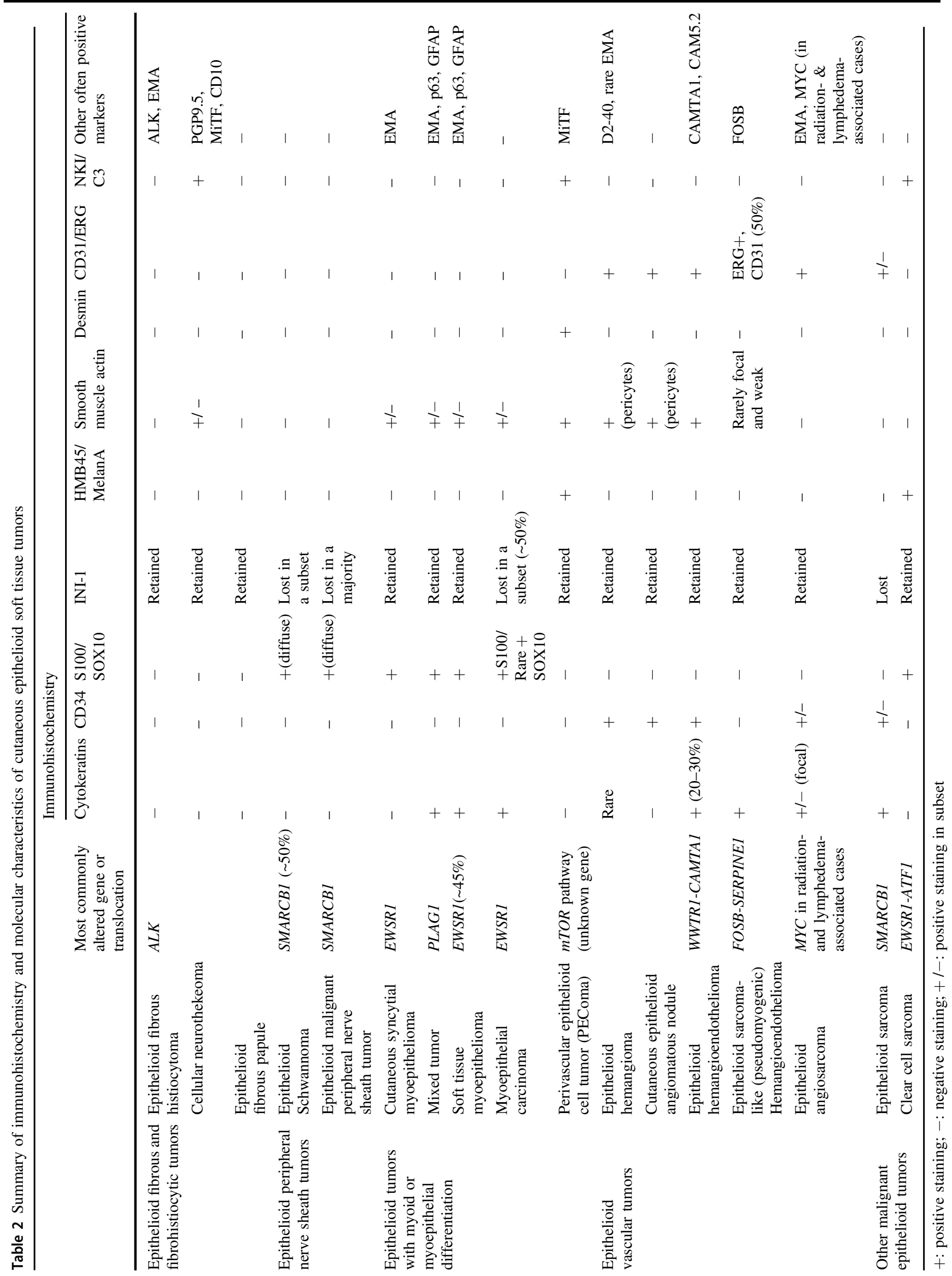


giant cells, perineural invasion, and lymphovascular invasion have all been reported in these tumors, but none of these features have been shown to affect the benign clinical course [7]. Immunohistochemically, the tumor cells show strong, diffuse expression of PGP9.5 and NKI/C3, both melanocytic markers of fairly low specificity, as well as focal or weak expression of MiTF in $60-80 \%$ of cases, and SMA in $10-50 \%$ of cases (Fig. 1f, g) [9]. They are negative for CD34, pancytokeratin, S100, SOX10, and desmin.

Given the lack of a specific immunohistochemical marker for these tumors, care should be taken to exclude entities in the differential diagnosis that are more aggressive, particularly melanocytic lesions given their common expression of NKI/C3. Melanocytic lesions can be excluded by demonstrating the tumor cells to be negative for $\mathrm{S} 100$ and S0X10. Plexiform fibrohistiocytic tumor, particularly the fibrohistiocytic and mixed types, can enter the differential diagnosis due to its nests and nodules of epithelioid or histiocytoid cells. These are locally aggressive tumors of intermediate malignant potential, and can be differentiated based on their prominent infiltrative growth, more extensive subcutaneous involvement, and typical biphasic mix of histiocytoid and fibroblastic components.

\section{Epithelioid fibrous papule (epithelioid angiofibroma)}

Epithelioid fibrous papule (epithelioid angiofibroma) most commonly presents as a dome-shaped papule on the nose or face of adults, which can be inadvertently suspected to be a papular melanocytic lesion or basal cell carcinoma [9]. These are benign lesions cured by simple excision or other localized modalities such as laser therapy, and are important to recognize in order to avoid overtreatment.

Morphologically, epithelioid fibrous papules resemble their conventional counterparts, which are dome-shaped lesions often demonstrating an epidermal collarette, but epithelioid variants consist of plump epithelioid stromal cells with abundant eosinophilic or clear cytoplasm within the superficial dermis (Fig. 1h) [10]. The lesion cellularity can vary widely, ranging from sheets of epithelioid cells often seen in the clear cell variant of fibrous papule, to relatively few epithelioid cells which more closely resemble a scattered histiocytic inflammatory pattern. Occasional multinucleation or bizarre nuclei can be present. The constituent cells of fibrous papule can predominantly or entirely contain clear cytoplasm or dense granular cytoplasm, denoted as clear cell and granular cell variants, respectively, but these variants show epithelioid cytomorphology as well.

The immunophenotype of the lesional cells is nonspecific, and the diagnosis can typically be made based on morphology on hematoxylin and eosin (H\&E)-stained sections, however, immunohistochemistry can aid in excluding morphologic mimics. The constituent cells in epithelioid fibrous papule are negative for melanocytic markers S100 protein, SOX10, HMB45, and Melan-A, which can be useful in excluding a melanocytic lesion. In examples with clear cell change, PAX-8 and cytokeratin negativity helps exclude metastatic clear cell renal cell carcinoma in a patient with a renal mass or prior history of renal cancer [9].

\section{Epithelioid peripheral nerve sheath tumors}

\section{Epithelioid schwannoma}

Epithelioid schwannomas are benign peripheral nerve sheath tumors usually arising in the dermis and superficial subcutis. They commonly present as superficial nodules in the extremities. Most are small $(<5 \mathrm{~cm})$, and they behave in a benign fashion.

Microscopically, epithelioid schwannomas are well circumscribed dermal or subcutaneous nodules surrounded by a thin, perineural capsule. Cells are arranged in sheets, nests, and cords, occasionally showing a trabecular pattern (Fig. 2a-c). By definition, more than 50\% of the constituent cells show epithelioid morphology, and while most cases are composed entirely of epithelioid cells, up to a third of cases contain a minor spindle cell component [11]. Similar to conventional schwannomas, hyalinized ectatic vessels are a common feature. Epithelioid schwannoma cells commonly contain irregular nuclei with reniform features or convoluted nuclear contours (Fig. 2b). Atypia is seen in up to a third of cases, but in the form of large, smudgy nuclei, which are considered to be degenerative in etiology. Cases with significant nuclear atypia (which has been defined in this entity as greater than 3:1 nuclear size variation between lesional cells) combined with a mitotic rate greater than 3 mitoses per 10 high power fields, which lack the necrosis, diffuse atypia, and infiltrative growth seen in epithelioid malignant peripheral nerve sheath tumors (MPNSTs) are considered "atypical variants." Thus far, this is considered a morphologic descriptor, as reported atypical epithelioid schwannomas with clinical follow-up demonstrate a benign clinical course identical to typical schwannomas.

Immunohistochemical features of epithelioid schwannomas include diffuse immunoreactivity for $\mathrm{S} 100$ protein and SOX10, and the true perineural capsule can be highlighted by EMA or GLUT1-positivivity within the perineural cells (Fig. 2d) [11-13]. Loss of INI1 expression is observed in nearly $50 \%$ of cases, correlating with inactivating mutations in the SMARCB1 gene (discussed in greater detail below under the epithelioid sarcoma section) [13, 14]. This finding is also seen in epithelioid MPNSTs, but in a much higher proportion of malignant cases. GFAP positivity is also seen in nearly half of cases, but epithelioid schwannomas are 
Fig. 2 Epithelioid peripheral nerve sheath tumors. a-d Epithelioid schwannomas. Epithelioid schwannomas are well circumscribed dermal or subcutaneous nodules surrounded by a thin, true perineural capsule (a). The constituent cells are epithelioid with reniform nuclei or convoluted nuclear contours, arranged in sheets and vague nests or clusters $(\mathbf{b}, \mathbf{c})$. Epithelioid schwannomas are diffusely positive for S100 (d). e-h Epithelioid malignant peripheral nerve sheath tumors (MPNSTs). Epithelioid MPNSTs are composed of infiltrative nodules of cells with large, vesicular nuclei with prominent nucleoli and frequent mitotic activity $(\mathbf{e}, \mathbf{f})$. Unlike conventional MPNSTs, epithelioid variants are typically diffusely positive for $\mathrm{S} 100$ (g). INI1 loss is observed in nearly all cases (h)
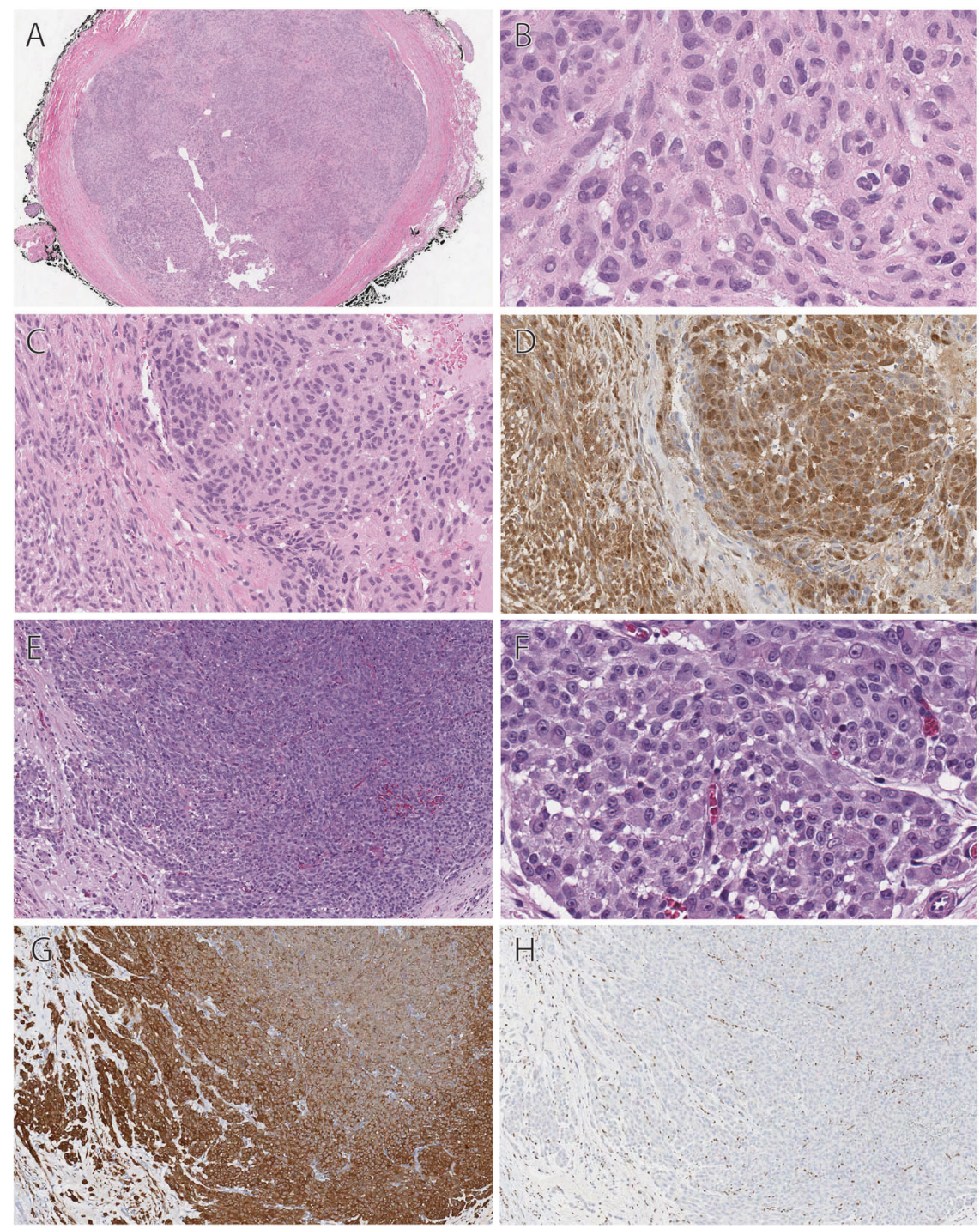

only rarely focally positive for keratins such as cytokeratin AE1/AE3, despite the cross-reactivity of the antibodies for cytokeratin AE1/AE3 with GFAP [13, 15].

Epithelioid schwannomas should be differentiated from both nodular melanomas and epithelioid MPNSTs. In addition to S100 protein and SOX10 expression, the vast majority of malignant melanomas also show positivity for melanocytic markers HMB45, Melan-A, and MiTF, in contrast to spindle cell/desmoplastic melanomas which often exhibit loss of one or more of these markers. Nodular melanomas also typically demonstrate a junctional component and have a higher mitotic rate. While there are no defined criteria separating atypical epithelioid schwannomas from epithelioid MPNSTs, the presence of atypical mitoses, necrosis, multinodular infiltrative growth, diffuse nuclear atypia or vesicular nuclei with prominent nucleoli all favor the diagnosis of epithelioid MPNST. Immunohistochemistry does not play a role in distinguishing epithelioid schwannoma from epithelioid MPNST, as both entities show diffuse expression of S100 protein and SOX10, and may or may not exhibit loss of INI1.

\section{Epithelioid malignant peripheral nerve sheath tumor}

Epithelioid MPNSTs are often superficial tumors, most commonly based in the subcutis of the lower extremities, but also can occur in the trunk and upper extremities. They are associated with a nerve or a component of epithelioid or conventional schwannoma or neurofibroma in $20 \%$ of cases, which is less common than their conventional counterparts [16]. Most epithelioid MPNSTs exhibit lobular growth with infiltrative borders. The constituent epithelioid cells exhibit high-grade cytologic features including large vesicular nuclei with prominent nucleoli and frequent mitoses, and more than one-fourth of tumors exhibit 
necrosis (Fig. 2e-h). The cells contain less cytoplasm compared to the lesional cells in epithelioid schwannomas, and the stroma more often exhibits at least focal myxoid change.

In contrast to conventional MPNST, epithelioid MPNSTs show diffuse and strong $\mathrm{S} 100$ protein positivity in nearly $90 \%$ of cases, exceeding its conventional spindle cell counterpart in both overall incidence and percentage of tumor cells within a given tumor, and also typically demonstrates diffuse and strong nuclear positivity for SOX10 (Fig. 2f) [14, 17]. Compared to conventional MPNSTs, epithelioid variants are much more likely to be wild-type rather than associated with neurofibromatosis type 1 (NF1). Almost all epithelioid MPNSTs show inactivating mutations of SMARCB1, which strongly correlates with loss of INI1 expression by immunohistochemistry (Fig. 2g) [14]. Recently, loss of trimethylation of lysine 7 of histone $\mathrm{H} 3$ (H3K27me3) by immunohistochemistry demonstrated in some conventional MPNSTs has been proposed as a useful diagnostic adjunct in the distinction of MPNST from benign nerve sheath tumors, however, almost all tested epithelioid variants of MPNSTs in several cohorts showed retained H3K27me3 [18-21].

Due to the diffuse S100 protein and SOX10 expression, the most critical diagnostic pitfall is melanoma, but epithelioid MPNSTs are invariably negative for the melanocytic markers HMB45, Melan-A (MART1), and MiTF. Rare cases of melanoma can also lack expression of these latter markers, and therefore it is critical to understand the clinical presentation and perform INI1 immunohistochemistry prior to making the diagnosis of epithelioid MPNST, as INI1 will be retained in melanoma. Epithelioid MPNSTs are also negative for cytokeratins and the more specific vascular markers CD31 and ERG, which are helpful in distinguishing epithelioid MPNST from carcinomas, epithelioid sarcomas, and epithelioid angiosarcomas, respectively. Distinction from atypical variants of epithelioid schwannoma is based on morphologic grounds, discussed above.

\section{Epithelioid tumors with myoid or myoepithelial differentiation}

\section{Cutaneous syncytial myoepithelioma}

Cutaneous syncytial myoepithelioma (CSM) presents as a papule or nodule involving the superficial dermis in the extremities of young adults and middle-aged adults, with a slight male predilection [22]. They are typically small (median diameter of $<1 \mathrm{~cm}$ ), and they behave in a benign fashion, only rarely recurring when incompletely excised.
Microscopically, these tumors consist of sheets of ovoid, histiocytoid, and spindled cells containing moderately abundant pale eosinophilic cytoplasm and uniformly bland, vesicular nuclei with inconspicuous nucleoli (Fig. 3a). The cells have poorly-defined cytoplasmic membranes, imparting a syncytial appearance, and variable amounts of myxoid stroma. Adipocytic metaplasia can be present in a subset of tumors (Fig. 3a) [23]. Lymphoid aggregates are commonly present, usually at the periphery of the nodule. Mitotic activity is low, and in most cases, it is challenging to identify any mitoses.

Immunophenotypically, EMA \& S100 are co-expressed in most cases, with GFAP, SMA, and p63 positivity being less common (Fig. 3b). Importantly, CSM lacks ALK expression, differentiating this tumor from epithelioid fibrous histiocytoma, with which it shares a significant proportion of clinical features.

Despite the more monotonous morphology and less common cytokeratin staining in CSM compared with cutaneous soft tissue myoepithelioma, there is genetic overlap in that up to $45 \%$ of soft tissue myoepitheliomas show EWSRl rearrangement. This contrasts with the myoepithelial-rich cutaneous neoplasm with ductal differentiation called cutaneous mixed tumor, also known as chondroid syringoma. Though these tumors are often lumped together with CSM and soft tissue myoepithelioma due to their shared immunophenotype and myoepithelialrich nature, most cutaneous mixed tumors actually share PLAG1 alterations with their salivary gland myoepithelialrich counterparts showing ductal differentiation, pleomorphic adenomas [24]. In CSM, the EWSRl gene is rearranged in approximately $80 \%$ of cases, which can be helpful in unusual cases lacking the classic immunophenotype [22]. Recent interrogation by RNA sequencing led to discovery that these tumors harbor a highly conserved fusion between exon 8 of EWSRl on chromosome $22 \mathrm{q}$ and exon 5 of $P B X 3$ on chromosome 9q $[25,26]$. Of note, at this time, the other myoepithelial neoplasms shown to harbor an identical EWSR1-PBX3 fusion are primary myoepitheliomas of bone [26, 27]. Among tumors in the differential diagnosis, an EWSR1 rearrangement should be sufficient in most challenging cases if the immunophenotype itself is not diagnostic [23].

\section{Mixed tumor and myoepithelioma}

Cutaneous mixed tumors (chondroid syringoma) and myoepitheliomas share features of myoepithelial differentiation and stromal characteristics, as well as many clinical features, but differ in that cutaneous mixed tumors by definition have at least a focal component of ductal differentiation, and they also have been shown to harbor genetic alterations similar to their salivary gland counterparts 
Fig. 3 Epithelioid tumors with myoid or myoepithelial differentiation. a, b Cutaneous syncytial myoepithelioma (CSM). CSMs are composed of sheet of uniform histiocytoid cells containing moderately abundant pale eosinophilic cytoplasm, bland nuclei, and poorly-defined cytoplasmic membranes (a). Adipocytic metaplasia can occasionally be seen $(\mathbf{a}$, right side of photomicrograph). EMA is positive in most cases (b). c-f Cutaneous myoepitheliomas. Cutaneous myoepitheliomas consist of epithelioid, plasmacytoid, and spindled cells arranged in corded, nested, and reticular patterns within a myxoid or chondromyxoid stroma (c). The tumor cells coexpress cytokeratin AE1/AE3 (d), S100 protein (e), and SOX10 (f) in most cases. Myoepithelial carcinomas show similar morphologic features to myoepitheliomas, but exhibit nuclear atypia with vesicular chromatin, prominent nucleoli, conspicuous mitotic activity including atypical mitoses, and necrosis $(\mathbf{g})$. Cutaneous PEComas consist of large epithelioid cells with clear or pale eosinophilic cytoplasm arranged in sheets, cords, and nests with an associated branching, capillary vasculature (h)
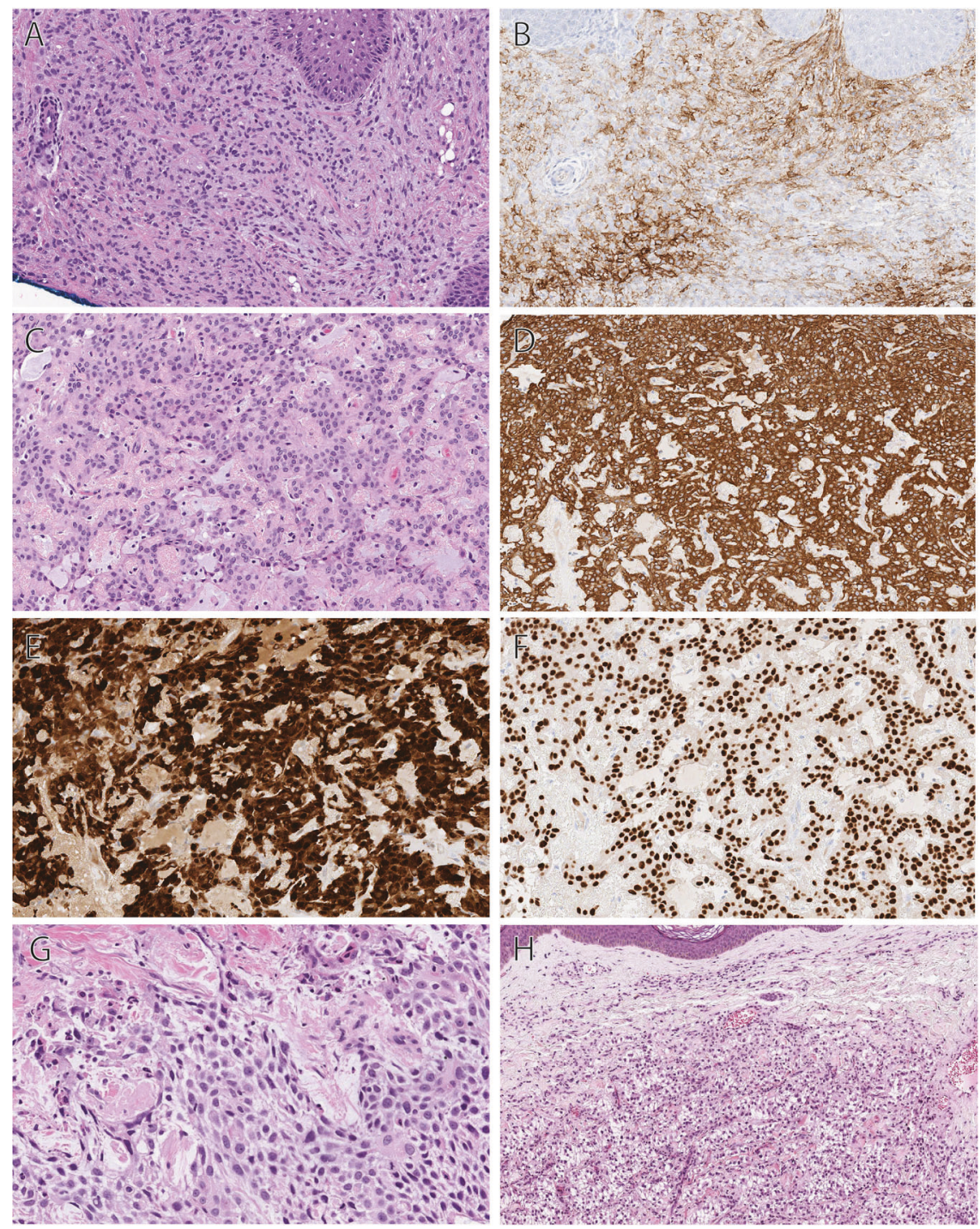

[28, 29]. Cutaneous mixed tumors occur as small, painless papules or nodules in the skin and subcutis, most commonly affecting the head and neck region or extremities. Cutaneous myoepitheliomas present in an identical fashion and in similar locations but show a higher predilection for the limb girdles. Both tumors are indolent and recur in $~ 20 \%$ of cases, typically after incomplete excision. Complete excision is therefore the mainstay of treatment and is often curative.

Morphologically, cutaneous mixed tumors are typically well circumscribed, unencapsulated nodules within the dermis, while cutaneous myoepitheliomas can more often show multilobation and extend into the underlying subcutis. They both consist of epithelioid, plasmacytoid, and spindled cells arranged in a variety of patterns within a chondroid, myxoid, chondromyxoid, or hyalinized stroma (Fig. 3c) $[9,28]$. Examples with predominantly rhabdoid morphology or clear cell change have also been described. The constituent cells contain amphophilic or eosinophilic cytoplasm and bland, ovoid nuclei with fine chromatin and small or inconspicuous nucleoli. These cells are arranged in a mix of patterns including corded, reticular, nested, and sheet-like, and while most tumors exhibit multiple patterns, a particular pattern can dominate in an individual tumor (Fig. 3c). Foci of osseous, cartilaginous, or adipocytic metaplasia can occur in a subset of cases, in both mixed tumors and myoepitheliomas. The morphologic feature that separates these tumors is the presence of ductal differentiation of either eccrine or apocrine type in cutaneous mixed tumors. Malignant mixed tumors are exceedingly rare, and malignant myoepitheliomas (myoepithelial carcinomas) are primarily designated as such by the presence of cytological atypia (discussed below). Mitotic activity and/or necrosis can both be present in benign myoepithelial tumors, but are not prominent features, and atypical mitoses are not seen in these tumors. 
Immunohistochemical analysis demonstrates both S100 protein and cytokeratin or EMA positivity in about $90 \%$ of cases (Fig. 3d, e) [30]. SOX10 positivity is also commonly expressed in both mixed tumors and myoepitheliomas $(\sim 80 \%)$, but is only present in a minor percentage of myoepithelial carcinomas (Fig. 3f) [12]. Other markers of myoepithelial differentiation including p63, GFAP, SMA, and calponin are also commonly positive, but in a lower proportion of tumors.

While cutaneous mixed tumors and myoepitheliomas are often grouped together as parts of a morphologic spectrum due to their myoepithelial differentiation, more recent developments in the underlying genetic alterations of these tumors suggest divergent etiologies. Mixed tumors commonly show gene rearrangements in PLAGl on chromosome $8 \mathrm{q} 12$, like salivary gland pleomorphic adenomas which share a similar phenotype. In addition to FISH interrogation for the PLAGl gene, nuclear staining for PLAG1 can also be detected by immunohistochemistry. The presence of PLAG1 alterations or stain positivity highly correlates with ductal differentiation in myoepithelial-rich tumors. PLAGl rearrangements seem to be mutually exclusive with EWSR1 rearrangements that are observed in a minority of cutaneous myoepitheliomas, seen in a much lower percentage of cases compared to cutaneous syncytial myoepithelioma. In myoepitheliomas with EWSRI rearrangement, partners include POU5F1, ZNF444, and ATF1, but the EWSR1-PBX3 rearrangement seen in cutaneous syncytial myoepitheliomas is not a typical fusion of conventional myoepitheliomas, other than examples arising in the bone [26, 27]. On rare occasion, rearrangements of FUS are observed in place of EWSRI.

\section{Myoepithelial carcinoma}

Cutaneous myoepithelial carcinomas share many of the morphologic and clinical features of myoepithelioma, arising in the extremities, limb girdles, and head and neck sites. They typically behave in a less aggressive fashion than their more common and better studied deep soft tissue counterparts, which have recurrence rates and metastatic rates of $\sim 40 \%$ each $[28,30]$.

Myoepithelial carcinomas morphologically resemble their benign counterparts, similarly consisting of sheets, nests, cords, and reticular patterns of mostly epithelioid or plasmacytoid cells, but are differentiated by their prominent cytological atypia, containing cells with large, irregular nuclei with coarse or vesicular chromatin and prominent nucleoli. Atypical nuclear features are the strongest correlate with aggressive behavior, and should be relied upon most heavily to distinguish from benign myoepithelial tumors (Fig. 3g) [28, 30]. However, necrosis, readily identifiable mitotic activity with atypical mitotic figures, and infiltrative borders are also significantly more common in myoepithelial carcinoma compared to myoepitheliomas, and while not independent adverse prognostic factors in and of themselves, they are supportive features of malignancy.

Like myoepitheliomas, myoepithelial carcinomas are also nearly always positive for a combination of cytokeratin AE1/AE3 or CAM5.2 and S100 protein, and variably positive for SMA, calponin, and GFAP. SOX10 is only positive in $\sim 30 \%$ of myoepithelial carcinomas, a significantly lower proportion than its benign counterpart [12]. EWSRI rearrangements are more commonly found in myoepithelial carcinomas than myoepitheliomas, seen in $\sim 50 \%$ of cases. Of note, loss of INI1 expression by immunohistochemistry is also seen in a small subset of myoepithelial carcinomas, however, these alterations are enriched in pediatric tumors with rhabdoid morphology, seen in nearly $50 \%$ of evaluated pediatric cases ( 9 out of 22 cases) [31, 32].

In addition to myoepitheliomas, the differential diagnosis for myoepithelial carcinoma also includes epithelioid sarcoma and epithelioid MPNST. Epithelioid sarcomas can be excluded by their lack of positivity for $\mathrm{S} 100$ protein, while epithelioid MPNSTs can be excluded by their lack of cytokeratin expression. INI1 loss is not helpful in making either distinction, since large subsets of each of these tumors demonstrate INI1 loss.

\section{Cutaneous perivascular epithelioid cell tumor}

Cutaneous perivascular epithelioid cell tumor (PEComa) most commonly presents in the deep soft tissues, but rare cases can be limited to the dermis primarily with only focal involvement of the subcutis. Primary cutaneous PEComas typically arise as single flesh-colored nodules or papules on the extremities of middle-aged adults, and thus far, reported cases typically behave in a benign fashion [33]. These findings are based on limited data, however, and complete excision is still therefore recommended in primary cutaneous cases.

Cutaneous PEComas are often poorly circumscribed and consist of large epithelioid cells with clear or pale eosinophilic cytoplasm arranged in sheets, cords, and nests with peripheral collagen trapping and a branching, capillary vasculature (Fig. 3h). The nuclear features are often bland with vesicular chromatin and small nucleoli. These tumors have a distinct immunophenotype, staining for melanocytic markers including Melan-A, HMB-45, MiTF, and $\mathrm{NKI} / \mathrm{C} 3$, as well as smooth muscle markers including SMA, calponin, and desmin. S100 protein is typically negative in the cells, differentiating from a melanocytic lesion with clear cytoplasm. Of these immunohistochemical stains, HMB45, MiTF, and NKI/ $\mathrm{C} 3$ are the most sensitive markers in primary cutaneous PEComas, each noted in $>90 \%$ of cases [34]. 
In contrast to deep soft tissue PEComas, which are often related to alterations of the TSC1/2 genes in the mTOR pathway and associated with tuberous sclerosis, primary cutaneous PEComas have not been shown to harbor alterations in TSC1/2. Similarly, they have not been found to be associated with TFE3 immunohistochemical expression or TFE3 gene alterations by FISH, as in a subset of soft tissue PEComas which typically lack smooth muscle marker positivity. Moreover, they do not show alterations in genes associated with melanocytic lesions, including $B R A F, G N A S$, $N R A S$, and KIT. Although a well-defined genetic alteration has not been consistently observed in primary cutaneous PEComas, Charli-Joseph et al. did find that 4EBP1, a downstream protein product within the mTOR pathway, was strongly expressed in nearly all of their tested cases, supporting the hypothesis that primary cutaneous PEComas still contain mTOR pathway alterations, albeit of a different sort than the TSC1/2 alterations often seen in soft tissue PEComas [34].

The differential diagnosis of primary cutaneous PEComas is broad and includes tumors with either smooth muscle or melanocytic differentiation. Cutaneous myoepithelial tumors with clear cell morphology can be distinguished by their expression of cytokeratins or EMA. Expression of these markers can also distinguish cutaneous metastases of carcinomas with clear cell morphology, such as metastatic renal cell carcinoma or hepatocellular carcinoma. Therefore, multiple negative cytokeratin markers are essential before reaching a diagnosis of cutaneous PEComa. Melanocytic lesions with clear cell morphology such as balloon cell nevi and melanoma can be distinguished by their lack of smooth muscle marker positivity and diffuse S100 protein expression. Cutaneous granular cell tumors are positive for S100 protein but lack expression of the more sensitive melanocytic markers for PEComa, including Melan-A, HMB-45, MiTF, and NKI/C3.

\section{Epithelioid vascular tumors}

\section{Epithelioid hemangioma}

Epithelioid hemangioma, also known as angiolymphoid hyperplasia with eosinophilia, most commonly arises within a vessel, often involving the dermis or subcutaneous tissues, particularly in the head and neck region around the ear. Clinically, they can recur in up to one-half of cases, but these are postulated to be due to residual or multifocal involvement of the native vessel as in other benign vascular processes.

Microscopically, epithelioid hemangiomas consist of a proliferation of well-formed small vessels and capillaries in a lobular pattern, often arising in association with a larger, thick-walled vessel (Fig. 4a). These small vessels are lined by plump endothelial cells with moderately abundant eosinophilic cytoplasm and ovoid or round vesicular nuclei with pinpoint nucleoli, imparting an epithelioid appearance, and due to their voluminous cytoplasm, they often show hobnail
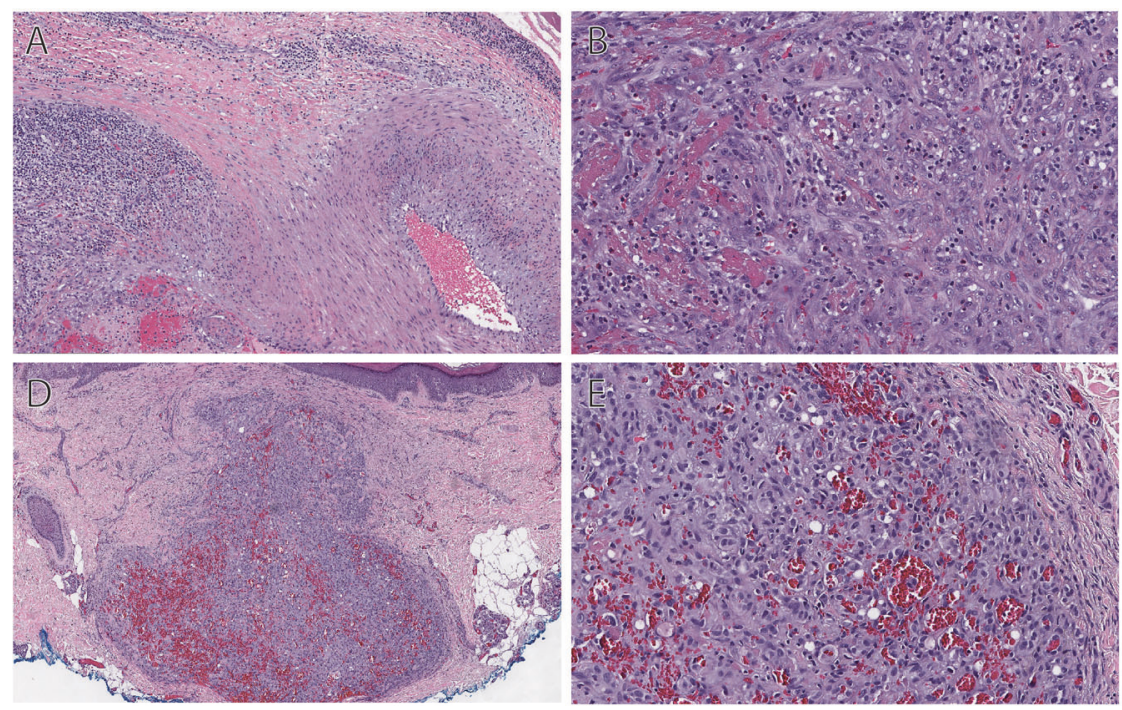

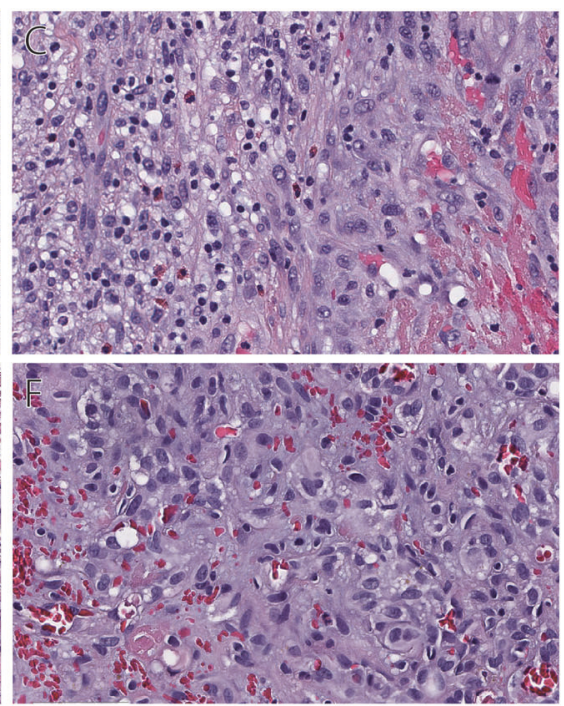

nodule (CEAN). CEAN is characterized by a circumscribed, superficial dermal nodule consisting of epithelioid cells with abundant eosinophilic cytoplasm arranged in diffuse sheets (d). Like epithelioid hemangioendothelioma, some cells contain intracytoplasmic vacuoles, but CEAN is differentiated by the presence of small well-formed vessels within the lesion, as well as the lack of a myxohyaline stromal matrix $(\mathbf{e}, \mathbf{f})$
Fig. 4 Benign epithelioid vascular tumors. a-c Epithelioid hemangioma. Epithelioid hemangioma consists of a proliferation of wellformed small vessels, often arising in association with a larger, thick-walled vessel (a). The small vessels are lined by endothelial cells with epithelioid morphology, containing abundant eosinophilic cytoplasm, and sometimes forming sheets (b). These tumors contain a mixed inflammatory infiltrate consisting of lymphocytes, plasma cells and eosinophils (c). d-f Cutaneous epithelioid angiomatous 
or "tombstone" morphology, projecting into the vascular lumens. In addition to composing well-formed capillaries, the endothelial cells can also form clusters and small sheets (Fig. 4b). A prominent inflammatory infiltrate is invariably present consisting of predominantly lymphocytes and eosinophils with scattered plasma cells and histiocytes; hence the original name for this entity (Fig. 4c).

Most importantly, epithelioid hemangiomas should be differentiated from low-grade malignant vascular neoplasms such as epithelioid hemangioendothelioma (EHE). These can also involve a large vessel, but in contrast, EHE usually lacks any significant degree of well-formed vascular channels and the epithelioid cells are embedded in a characteristic myxohyaline matrix, often showing intracytoplasmic vacuolization. In borderline cases, positive CAMTA1 by immunohistochemistry or FISH can be a helpful diagnostic adjunct in the confirmation of EHE [35].

\section{Cutaneous epithelioid angiomatous nodule}

Cutaneous epithelioid angiomatous nodule is usually a small $(<1 \mathrm{~cm})$ papule or nodule arising on the extremities, trunk or head and neck of middle-aged individuals, but can arise across a broad age range, and can also occasionally present in clusters of lesions.

Histologically, these lesions are characterized by a circumscribed, superficial dermal nodule consisting of epithelioid cells with abundant eosinophilic cytoplasm arranged in diffuse sheets in association with scattered small well-formed vessels (Fig. 4d) [36]. The cells lack any significant nuclear atypia, containing large, ovoid vesicular nuclei with smooth nuclear contours, evenly marginated chromatin, and conspicuous nucleoli, and mitotic activity is often noted but remains low. Scattered cells can contain intracytoplasmic vacuoles, and the stromal component is scant, composed of intervening bands of dermal collagen (Fig. 4f, g). Immunophenotypically, the epithelioid cells stain with vascular markers CD31, CD34, and ERG. Some authors purport that cutaneous epithelioid angiomatous nodule represents a solid, superficial variant of epithelioid hemangioma due to its overlapping cytomorphology and benign nature [37].

The differential diagnosis of cutaneous epithelioid angiomatous nodule includes epithelioid hemangioma, in which classic examples shows a much greater degree of vascular channel formation with similar bland cytologic features of the epithelioid cells, and often more prominent inflammatory components and multilobularity. Epithelioid angiosarcoma can be differentiated by its much greater degree of nuclear pleomorphism, high mitotic rate (often exceeding 10 per 10 high power fields), and infiltrative borders. Epithelioid sarcoma-like (pseudomyogenic) hemangioendothelioma is less cellular, less circumscribed, and contains cells with more abundant, intense eosinophilic cytoplasm and a slightly greater degree of nuclear atypia, and the tumor cells also show positivity for cytokeratins and FOSB.

\section{Epithelioid hemangioendothelioma}

Epithelioid hemangioendotheliomas (EHE) are low-grade malignant vascular tumors which most often affect middleaged individuals but can affect adults of all ages. When EHE involves the skin, it is often by extension or metastasis from the deeper soft tissues or underlying bone, and this should be excluded when cutaneous involvement is documented. Primary cutaneous examples usually arise as solitary lesions within the subcutis of the extremities, often in association with a vessel, and can extend superficially to involve the dermis [35].

Microscopically, EHE consists of a variably cellular proliferation of epithelioid cells arranged in cords and vague lobules, embedded within a myxohyaline stroma (Fig. 5a, b). The cells contain moderately abundant pale eosinophilic cytoplasm with frequent single intracytoplasmic vacuoles, and predominantly exhibit bland nuclear features with vesicular chromatin and inconspicuous nucleoli but can occasionally show more striking scattered nuclear enlargement and pleomorphism. These tumors are typically well circumscribed when primary in the skin, but can be multinodular are poorly circumscribed on occasion [38]. Overlying epidermal acanthosis is a common feature.

Primary cutaneous EHE shows a similar immunophenotype to EHE of bone and visceral organs, as they are positive for CD31, CD34, ERG, and SMA (Fig. 5c) [38]. Low-molecular weight cytokeratins such as CAM5.2 can be expressed in some cases, but the tumor cells are negative for cytokeratin AE1/AE3 and EMA.

EHEs have been found to harbor a recurrent fusion between WWTRl on chromosome 3q23-24 and CAMTAl on chromosome $1 \mathrm{p} 36$ resulting in a $\mathrm{t}(1 ; 3)(\mathrm{p} 36 ; \mathrm{q} 23-25)$ translocation in $\sim 90 \%$ of cases. Rare examples (fewer than $5 \%$ ) with YAP1-TFE3 fusions have been described, though this fusion has yet to be described in primary cutaneous EHE [39-41]. CAMTA1 fusions correlates with nuclear CAMTA1 expression by immunohistochemistry in a similar proportion of cases, particularly when excluding tumors with TFE3 alterations [42].

EHE is considered a vascular neoplasm of intermediate malignant potential with metastatic rates ranging from 20 to $30 \%$. Primary cutaneous EHE is significantly rarer, but based on existing reported cases, these tumors appear to have a better prognosis than their deep soft tissue, bone, and visceral organ counterparts, with most cases behaving in an indolent fashion. A proposal for risk stratification of EHE has been made on the basis of mitotic rate and size, which has not been validated in a cohort of primary cutaneous 
Fig. 5 Malignant epithelioid vascular tumors. a-c Epithelioid hemangioendothelioma (EHE). EHE is a poorly circumscribed proliferation of epithelioid cells arranged in cords, sheets, and vague lobules within a myxohyaline stroma (a). The cells contain moderately abundant pale eosinophilic cytoplasm with frequent single intracytoplasmic vacuoles, and lack well-formed vessels (b).

The cells are uniformly nuclear positive for ERG $(\mathbf{c})$. $\mathbf{d}-\mathbf{f}$ Epithelioid sarcoma-like (pseudomyogenic) hemangioendothelioma (ESLH). ESLH consists of sheets and vague fascicles of epithelioid and spindled cells with abundant eosinophilic cytoplasm, imparting a smooth muscle morphology (d). These tumors lack well-formed vascular channels, but co-express cytokeratin AE1/AE3 (e) and ERG (f). g, h Epithelioid angiosarcomas. Epithelioid angiosarcomas consist of infiltrative sheets and nodules of epithelioid cells with large, round vesicular nuclei with prominent nucleoli and frequent mitotic activity (g). The tumor cells are diffusely positive for CD31 (h), as well as ERG
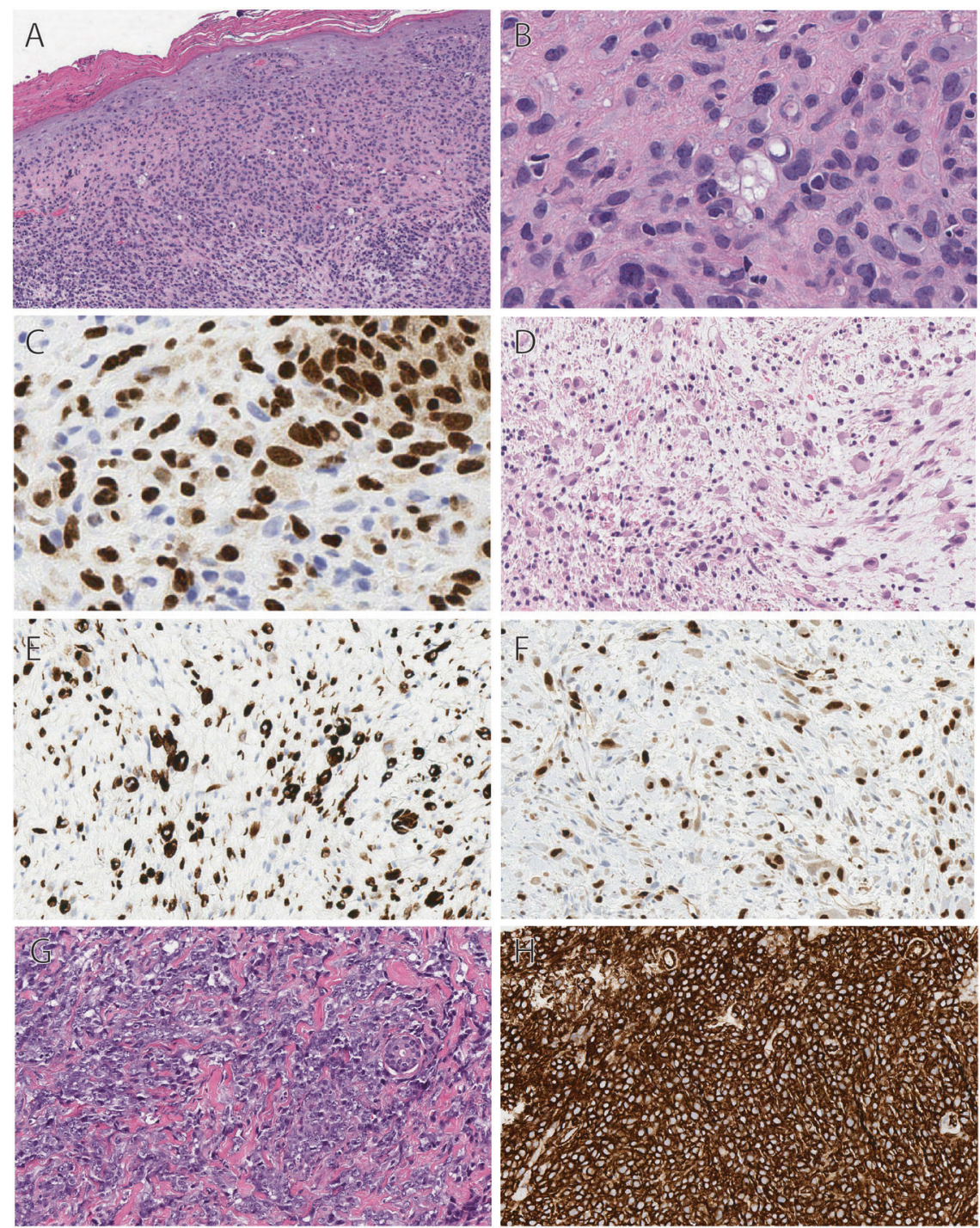

EHE, however, most reported cases of primary cutaneous EHE would fall into the lower-risk profile [43].

The differential diagnosis of EHE includes both epithelioid sarcoma and epithelioid sarcoma-like hemangioendothelioma. EHE lacks the central necrotizing granulomalike pattern of necrosis seen in epithelioid sarcoma. Epithelioid sarcoma can be further distinguished by its EMA and cytokeratin expression, loss of INI1 expression, and lack of CAMTA1 positivity. Epithelioid sarcoma-like hemangioendothelioma shows similar expression of endothelial markers CD31 and ERG but is differentiated by its positivity for cytokeratin and FOSB, in addition to retained INI1 expression.

\section{Epithelioid sarcoma-like (pseudomyogenic) hemangioendothelioma}

Epithelioid sarcoma-like hemangioendotheliomas most commonly arise subcutaneously within the lower extremities of young adults and adolescents, affecting a mean age of $\sim 30$ years, and disproportionally affecting male patients. These tumors exhibit a high incidence of multifocality, and multifocal tumors can present in both deep and superficial soft tissues, as well as bone [44]. As a result, approximately half of all tumors arise in the dermis and subcutis. Recurrences are common, but metastases have only been rarely reported; thus, this entity is considered to be a vascular tumor of low to intermediate malignant potential.

Morphologically, these tumors are often poorly circumscribed and consist of sheets, nests, or vague fascicles of cells within a desmoplastic stroma, which show immunophenotypic evidence of endothelial differentiation but lack well-formed vascular channels $[44,45]$. While many of the cells show epithelioid morphology with vesicular chromatin and prominent nucleoli, in most tumors a spindled cell morphology is dominant, albeit with abundant eosinophilic cytoplasm (Fig. 5d). A common and helpful diagnostic feature is the presence of a predominantly or exclusively 
neutrophilic inflammatory cell infiltrate. Mitotic activity is typically quite low, with a median of 1 per 10 high power fields, and necrosis is usually absent.

Immunophenotypically, these tumors co-express cytokeratin AE1/AE3 and vascular markers CD31 and ERG, while they commonly lack positivity for CD34 and MNF116 (Fig. 5e, f). Despite their cytoplasmic quality imparting morphologic characteristics of smooth muscle cells, myogenic markers including SMA, calponin, desmin, and myogenin are negative or only focal and weakly immunoreactive [44]. Importantly, these tumors show retained expression of INI1, a feature useful in distinguishing from epithelioid sarcoma.

A unique balanced translocation was found among these tumors resulting in a fusion between $F O S B$ at $7 \mathrm{q} 22$ and SERPINE1 at $19 \mathrm{q} 13$, resulting in a high level expression of the transcription factor product of FOSB [46]. More recently, a new antibody against FOSB protein has been shown to be both sensitive and specific among tumors with vascular differentiation, with epithelioid sarcoma-like hemangioendotheliomas demonstrating diffuse and strong nuclear expression [47, 48].

\section{Epithelioid angiosarcoma}

Epithelioid angiosarcoma is a morphologic variant of angiosarcoma which has been associated with poorer prognosis compared to angiosarcomas without epithelioid features [49]. A lower overall percentage of cutaneous angiosarcomas show prominent epithelioid morphology compared to deep soft tissue counterparts. Nevertheless, epithelioid angiosarcomas do occur in the dermis and subcutis, and require a high index of suspicion in order to avoid misdiagnosis as carcinoma.

Epithelioid angiosarcoma most commonly presents as infiltrative sheets and nodules of epithelioid cells with scant eosinophilic cytoplasm and high-grade nuclear features including large, round vesicular nuclei with prominent nucleoli and frequent mitotic activity (Fig. 5g). Vasoformation is usually less conspicuous compared to its nonepithelioid counterpart, and thus these tumors are more likely to show histologic overlap with carcinomas and melanomas, however, intratumoral hemorrhage is often a feature and can be a helpful diagnostic clue [35]. In tumors that contain more well-formed vascular channels, they are usually better seen at the periphery of the lesion. By immunohistochemistry, CD31 and ERG are highly sensitive for epithelioid angiosarcomas, while the use of CD34 as a confirmatory marker for angiosarcoma should be avoided, as a high proportion of high-grade angiosarcomas can be negative for CD34 (Fig. 5h). At least focal EMA and cytokeratin expression (particularly low-molecular weight cytokeratins) is common among epithelioid angiosarcomas, and presents a diagnostic pitfall with both epithelioid sarcoma and carcinomas [36, 50].

Owing to its high-grade nuclear features, the differential diagnosis of cutaneous epithelioid angiosarcoma primarily includes poorly differentiated carcinomas, high-grade lymphomas, and melanoma. Due to the common positivity for epithelial markers such as cytokeratins and EMA, these markers are not useful in distinguishing angiosarcomas from carcinomas. Thus, when encountering a cutaneous epithelioid neoplasm with prominent atypia, an endothelial marker such as ERG or CD31 should be added to the typical screening panel of cytokeratins, CD34, S100, SMA, and CD45. Expression of either endothelial marker will also help to exclude high-grade lymphomas and melanoma.

\section{Other malignant epithelioid soft tissue tumors}

\section{Epithelioid sarcoma}

Epithelioid sarcoma is a malignant soft tissue tumor of unknown histogenesis, with tumor cells demonstrating phenotypic evidence of epithelial differentiation. Clinically, these tumors typically involve the distal upper extremities of young adults and adolescent individuals with a median age of 25 years, most commonly involving the dermis, subcutis, and aponeuroses or tendons, and affecting males twice as often as females [51]. However, ES can occur in virtually any location, with rare cases reported involving the face and genital area. These tumors have a propensity for multifocality at initial presentation, forming multiple adjacent ulcerated firm nodules. In addition, they demonstrate high rates of local recurrence $(>50 \%)$ and regional metastasis (just $<50 \%$ ), often forming new tumors within the same extremity in a location proximal to the initial tumor site [51, 52]. In addition to tumors arising in the distal extremities, which is known as "classic-type" epithelioid sarcoma, a less common "proximal-type" is also described that most commonly occurs in the deep soft tissues of the limb girdles and proximal lower extremities [53]. The proximal-type of ES shares a similar underlying immunohistochemical profile and genetic alteration, but shows higher grade histologic features. Occasionally, the proximal-type of ES can involve the dermis and subcutis primarily, though cutaneous involvement by proximal-type ES more commonly occurs in recurrences or regional metastases. Since their initial description, underlying alterations in the genes encoding proteins in the SWI/SNF chromatin-remodeling complex have been described in most cases.

Histologically, epithelioid sarcomas form single or multiple poorly circumscribed nodules of epithelioid tumor 
cells with moderately abundant pale eosinophilic cytoplasm and a prominent central zone of necrosis (Fig. 6a-d). In classic-type ES, the cells typically show only mild cytologic atypia, with moderately sized vesicular nuclei containing small or inconspicuous nucleoli, showing only occasional mitoses (Fig. 6b). Infiltrative cords and nests of epithelioid cells extending beyond the main nodules are common and may to some degree account for the high rates of local recurrence. The low power pattern of central necrosis surrounded by palisaded epithelioid cells can mimic necrotizing granulomatous inflammation or necrobiotic granulomatous lesions such as rheumatoid nodule or necrobiosis lipoidica. Based on this common feature, a high index of suspicion should be maintained for ES when granulomatous inflammation is seen in the extremities of young adults. The proximal-type of ES consists of cells with much larger, pleomorphic vesicular nuclei with prominent nucleoli, abundant mitotic activity, and frequent rhabdoid morphology (Fig. 6e-f). Similar to classic-type ES, proximal-type ES also shows central necrosis, but is more likely to demonstrate a peritheliomatous pattern of geographic necrosis owing to its greater proliferation rate, and therefore does not exhibit significant morphologic overlap with nodular granulomatous inflammation (Fig. 6e) [53]. While the degree of cytokeratin expression is slightly lower on average in proximal-type ES, the distinction between classic-type and proximal-type ES is largely made based on morphology with consideration of anatomic location.

By Immunohistochemistry, both classic-type and proximal-type show at least focal, and most often diffuse, expression of both high- and low-molecular weight cytokeratins, as well as EMA [54]. More specifically, the tumor cells are commonly diffusely or predominantly positive for EMA, cytokeratins AE1/AE3 and CAM5.2 (keratin 8 and 18 ) and at least focally positive for cytokeratin $34 \beta \mathrm{E} 12$
Fig. 6 Other epithelioid malignant neoplasms of uncertain histogenesis. $\mathbf{a}-\mathbf{d}$ Classic-type epithelioid sarcoma (ES). Classic-type ES consists of vague nodules of epithelioid tumor cells with moderately abundant pale eosinophilic cytoplasm and mild cytologic atypia, with a central zone of necrosis which mimics necrobiotic granulomatous lesions from lower power magnification $(\mathbf{a}, \mathbf{b})$. The epithelioid cells are positive for EMA (c) and show nuclear loss of INI1 (d). e, f Proximal-type ES often shows a greater degree of coagulative-type necrosis and consists of sheets of larger cells with vesicular chromatin and prominent nucleoli $(\mathbf{e}, \mathbf{f})$ but show a similar

immunohistochemical profile to classic-type ES. g, h Cutaneous clear cell sarcoma (CCS). CCS consists of nests of plump epithelioid and spindled cells containing abundant clear or pale eosinophilic cytoplasm and uniform ovoid nuclei with vesicular chromatin and prominent nucleoli (g). Thick, hyalinized collagen bundles separate the nests (h), which can be a useful morphologic feature to distinguish from melanoma
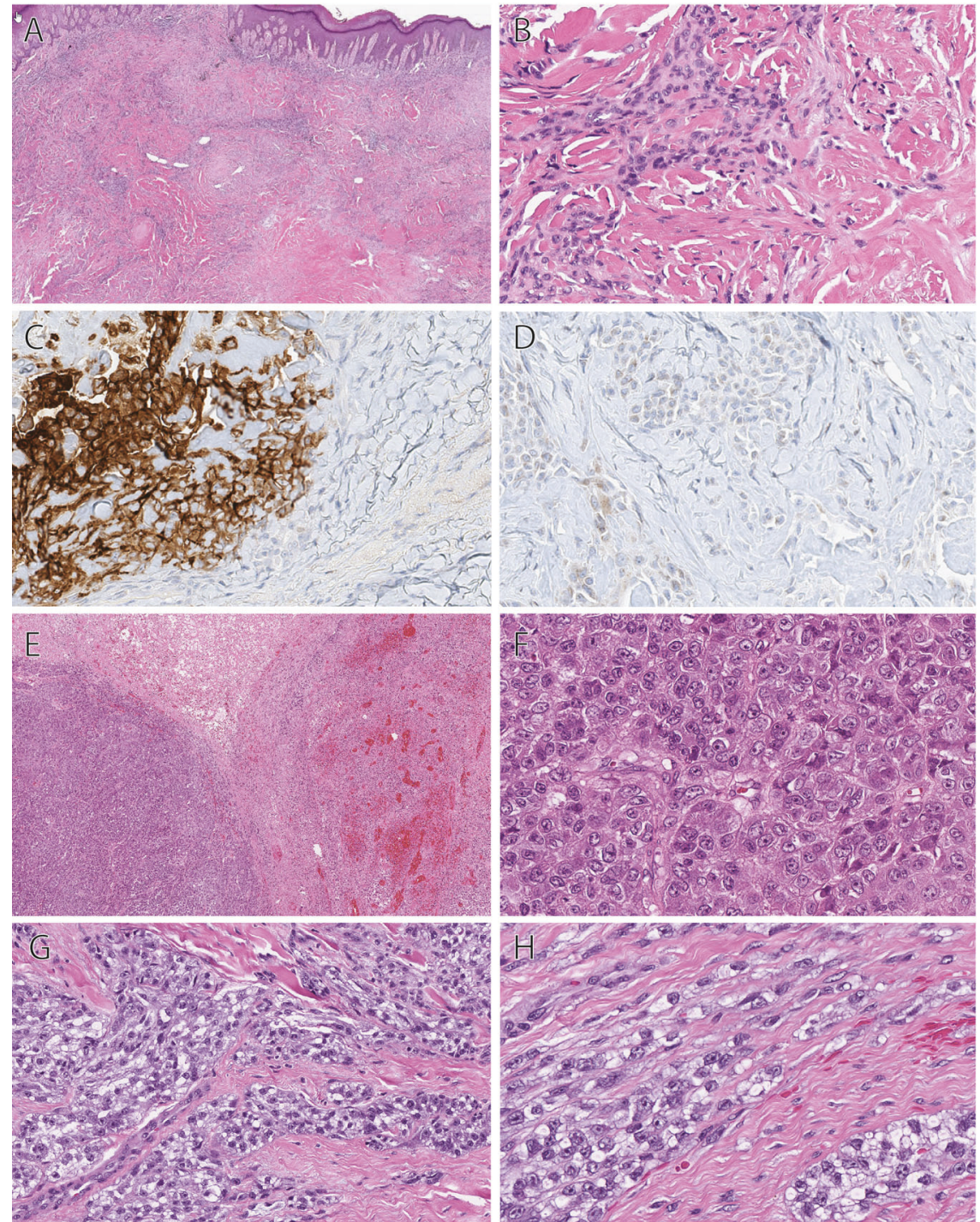
(keratins 1, 5, 10, and 14/15), while they are rarely positive for CK7 and are negative for CK20 (Fig. 6c) [54, 55]. The presence of cytokeratin expression helps to exclude necrobiotic granulomatous lesions. CD34 and SMA are positive in about half of cases, and until the molecular alterations in ES were elucidated, CD34 was useful in distinguishing these tumors from metastatic carcinomas. S100 protein and CD31 are frequently negative in ES, differentiating them from myoepithelial carcinomas and malignant vascular tumors, respectively.

Though ES typically harbor complex karyotypes and high copy number variations, a unifying oncogenic driver mutation of deletions in the SMARCB1 gene was discovered after initial reports of translocations involving chromosome 22q11 [56-58, 44, 52-54]. This gene encodes INI1, a ubiquitously expressed subunit of the SWI/SNF chromatin-remodeling complex, and thus SMARCBI is considered as a tumor suppressor gene which has also been implicated as the molecular driver in malignant rhabdoid tumors, central nervous system atypical teratoid/rhabdoid tumors, and the epithelioid malignant peripheral nerve sheath tumors and myoepithelial neoplasms described above. This discovery has become very useful diagnostically, as a lack of INI1 expression by immunohistochemistry is seen in approximately $90 \%$ of both classic and proximal-type ES (Fig. 6d) [32].

\section{Clear cell sarcoma}

Clear cell sarcoma (CCS) of tendons and aponeuroses are primary soft tissue sarcomas which, like epithelioid sarcomas, involve the distal extremities of young adults and adolescents, albeit with a slight female predominance. While typically a deep-seated soft tissue tumor, rarely these cases can be primary to the dermis and superficial subcutis, which can be quite confounding diagnostically due to significant morphologic and immunophenotypic overlap of CCS with malignant melanoma [59-62]. Despite overlapping features, it is important to distinguish these entities due to a significant difference in effective treatments. Fortunately, a high index of suspicion for CCS based on clinical features and subtle morphologic features such as a nested architecture, low mitotic activity, and uniform cytology, can prompt the surgical pathologist to perform molecular analysis for a recurrent genetic alteration in these tumors.

Microscopically, CCS shares some features with the clear cell variant of malignant melanoma, consisting of a nested or fascicular proliferation of plump spindled and epithelioid cells with abundant clear or pale eosinophilic cytoplasm. The constituent cells contain ovoid nuclei with vesicular chromatin and prominent nucleoli, but the cells typically show uniform nuclear features in contrast to malignant melanoma (Fig. 6g). Thick, hyalinized collagen bundles separate the nests and fascicles, which is one of the most helpful morphologic features to distinguish CCS from melanoma (Fig. 6h) [59]. Though primary cutaneous CCS can show a close relationship with the epidermis mimicking a junctional component at the dermal-epidermal junction, pagetoid involvement of the epidermis has not been described in CCS, and when present supports a diagnosis of melanoma [60].

Immunophenotypically, CCS commonly expresses markers of melanocytic differentiation including S100 protein, SOX10, HMB45, Melan-A, and NKI/C3. All of these markers are commonly expressed in melanoma as well, though less consistent HMB45 and Melan-A expression is seen in melanomas with spindle cell features. In addition, these tumors show melanocytic differentiation at the ultrastructural level, with melanosomes filling the cytoplasm when analyzed by electron microscopy.

Given the histologic, immunophenotypic, and even ultrastructural overlap between CCS and melanoma, particularly CCS involving the dermis, molecular studies can be a helpful diagnostic aid in distinguishing these two entities. Most tested cases of CCS have been shown to harbor the recurrent genetic alteration $\mathrm{t}(12 ; 22)(\mathrm{q} 13 ; \mathrm{q} 12)$ resulting from the fusion of EWSRI at 22q12 with ATF1 at 12q13. Less commonly, EWSR1 forms a fusion with CREB1 at 2q13. In clinically or morphologically suspected cases, reverse transcriptase polymerase chain reaction (PCR) can be used to detect these two fusions, or alternatively, break-apart FISH for EWSR1. Importantly, alterations in EWSRI have not been observed in melanoma, and the reciprocal is true as well, as alterations of genes involved in the MAPK pathway observed in most melanomas (such as BRAF and NRAS) are not seen in CCS.

\section{Conclusions}

By virtue of their relatively low incidence, epithelioid cutaneous soft tissue tumors should often be considered a diagnosis of exclusion, with carcinomas, melanocytic lesions, and even lymphomas considered in the differential diagnosis. Based on this premise, when considering the diagnosis of an epithelioid cutaneous soft tissue tumor, an initial limited immunohistochemical panel is recommended, including a broad-spectrum cytokeratin stain, CD34, S100 or SOX10, SMA, and CD45, with consideration of CD31 or ERG in high-grade tumors. In most cases, this initial panel should help to stratify a lesion into the outlined differentiation schema, and additional morphologic features such as stromal changes and atypia can guide further ancillary studies if necessary. This approach will help avoid the potentially disastrous consequences of misdiagnosing a dermal metastasis from an unrecognized melanoma or 
primary carcinoma, leading to more appropriate treatment and better outcomes. As molecular pathology advances in its diagnostic utility and economic accessibility, distinctions between these tumors will become less challenging, but as of yet, morphology and immunohistochemical analysis still play a vital role in appropriate management of these tumors, with molecular analysis serving as a useful adjunct in difficult or unusual cases.

\section{Compliance with ethical standards}

Conflict of interest The authors declare that they have no conflict of interest.

Publisher's note Springer Nature remains neutral with regard to jurisdictional claims in published maps and institutional affiliations.

\section{References}

1. Jedrych J, Nikiforova M, Kennedy TF, Ho J. Epithelioid cell histiocytoma of the skin with clonal ALK gene rearrangement resulting in VCL-ALK and SQSTM1-ALK gene fusions. Br J Dermatol. 2015;172:1427-9.

2. Doyle LA, Marino-Enriquez A, Fletcher CD, Hornick JL. ALK rearrangement and overexpression in epithelioid fibrous histiocytoma. Mod Pathol. 2015;28:904-12.

3. Dickson BC, Swanson D, Charames GS, Fletcher CD, Hornick JL. Epithelioid fibrous histiocytoma: molecular characterization of ALK fusion partners in 23 cases. Mod Pathol. 2018;31:753-62.

4. Kazakov DV, Kyrpychova L, Martinek P, Grossmann P, Steiner $\mathrm{P}$, Vanecek T, et al. ALK gene fusions in epithelioid fibrous histiocytoma: a study of 14 cases, with new histopathological findings. Am J Dermatopathol. 2018;40:805-14.

5. Wiesner T, He J, Yelensky R, Esteve-Puig R, Botton T, Yeh I, et al. Kinase fusions are frequent in Spitz tumours and spitzoid melanomas. Nat Commun. 2014;5:3116.

6. Fetsch JF, Laskin WB, Hallman JR, Lupton GP, Miettinen M. Neurothekeoma: an analysis of 178 tumors with detailed immunohistochemical data and long-term patient follow-up information. Am J Surg Pathol. 2007;31:1103-14.

7. Hornick JL, Fletcher CD. Cellular neurothekeoma: detailed characterization in a series of 133 cases. Am J Surg Pathol. 2007;31:329-40.

8. Zedek DC, White WL, McCalmont TH. Desmoplastic cellular neurothekeoma: clinicopathological analysis of twelve cases. J Cutan Pathol. 2009;36:1185-90.

9. Billings SD, Patel RM, Buehler D. Soft tissue tumors of the skin. Springer Science+Business Media; 2019.

10. Kucher C, McNiff JM. Epithelioid fibrous papule - a new variant. J Cutan Pathol. 2007;34:571-5.

11. Hart J, Gardner JM, Edgar M, Weiss SW. Epithelioid schwannomas: an analysis of 58 cases including atypical variants. Am J Surg Pathol. 2016;40:704-13.

12. Miettinen M, McCue PA, Sarlomo-Rikala M, Biernat W, Czapiewski $\mathrm{P}$, Kopczynski J, et al. Sox10-a marker for not only schwannian and melanocytic neoplasms but also myoepithelial cell tumors of soft tissue: a systematic analysis of 5134 tumors. Am J Surg Pathol. 2015;39:826-35.

13. Jo VY, Fletcher CDM. SMARCB1/INI1 loss in epithelioid schwannoma: a clinicopathologic and immunohistochemical study of 65 cases. Am J Surg Pathol. 2017;41:1013-22.
14. Schaefer IM, Dong F, Garcia EP, Fletcher CDM, Jo VY. Recurrent SMARCB1 inactivation in epithelioid malignant peripheral nerve sheath tumors. Am J Surg Pathol. 2019;43:835-43.

15. Fanburg-Smith JC, Majidi M, Miettinen M. Keratin expression in schwannoma; a study of 115 retroperitoneal and 22 peripheral schwannomas. Mod Pathol. 2006;19:115-21.

16. Jo VY, Fletcher CD. Epithelioid malignant peripheral nerve sheath tumor: clinicopathologic analysis of 63 cases. Am J Surg Pathol. 2015;39:673-82.

17. Rekhi B, Kosemehmetoglu K, Tezel GG, Dervisoglu S. Clinicopathologic features and immunohistochemical spectrum of 11 cases of epithelioid malignant peripheral nerve sheath tumors, including INI1/SMARCB1 results and BRAF V600E analysis. APMIS. 2017;125:679-89.

18. Cleven AH, Sannaa GA, Briaire-de Bruijn I, Ingram DR, van de Rijn M, Rubin BP, et al. Loss of H3K27 tri-methylation is a diagnostic marker for malignant peripheral nerve sheath tumors and an indicator for an inferior survival. Mod Pathol. 2016;29:582-90.

19. Otsuka H, Kohashi K, Yoshimoto M, Ishihara S, Toda Y, Yamada Y, et al. Immunohistochemical evaluation of H3K27 trimethylation in malignant peripheral nerve sheath tumors. Pathol Res Pract. 2018;214:417-25.

20. Prieto-Granada CN, Wiesner T, Messina JL, Jungbluth AA, Chi $\mathrm{P}$, Antonescu CR. Loss of $\mathrm{H} 3 \mathrm{~K} 27 \mathrm{me} 3$ expression is a highly sensitive marker for sporadic and radiation-induced MPNST. Am J Surg Pathol. 2016;40:479-89.

21. Asano N, Yoshida A, Ichikawa H, Mori T, Nakamura M, Kawai A, et al. Immunohistochemistry for trimethylated H3K27 in the diagnosis of malignant peripheral nerve sheath tumours. Histopathology. 2017;70:385-93.

22. Jo VY, Antonescu CR, Zhang L, Dal Cin P, Hornick JL, Fletcher CD. Cutaneous syncytial myoepithelioma: clinicopathologic characterization in a series of 38 cases. Am J Surg Pathol. 2013; 37:710-8.

23. Alomari AK, Brown N, Andea AA, Betz BL, Patel RM. Cutaneous syncytial myoepithelioma: a recently described neoplasm which may mimic nevoid melanoma and epithelioid sarcoma. J Cutan Pathol. 2017;44:892-7.

24. Jo VY, Fletcher CD. Myoepithelial neoplasms of soft tissue: an updated review of the clinicopathologic, immunophenotypic, and genetic features. Head Neck Pathol. 2015;9:32-8.

25. Jo VY, Antonescu CR, Dickson BC, Swanson D, Zhang L, Fletcher CDM, et al. Cutaneous syncytial myoepithelioma is characterized by recurrent EWSR1-PBX3 fusions. Am J Surg Pathol. 2019;43:1349-54.

26. Agaram NP, Chen HW, Zhang L, Sung YS, Panicek D, Healey $\mathrm{JH}$, et al. EWSR1-PBX3: a novel gene fusion in myoepithelial tumors. Genes Chromosomes Cancer. 2015;54:63-71.

27. Yun S, Kim SH, Cho HS, Choe G, Lee KS. EWSR1-PBX3 fused myoepithelioma arising in metatarsal bone: case report and review of the literature. Pathol Int. 2019;69:42-7.

28. Jo VY. Myoepithelial tumors: an update. Surg Pathol Clin. 2015; 8:445-66.

29. Mentzel T, Requena L, Kaddu S, Soares de Aleida LM, Sangueza OP, Kutzner H. Cutaneous myoepithelial neoplasms: clinicopathologic and immunohistochemical study of 20 cases suggesting a continuous spectrum ranging from benign mixed tumor of the skin to cutaneous myoepithelioma and myoepithelial carcinoma. J Cutan Pathol. 2003;30:294-302.

30. Hornick JL, Fletcher CD. Myoepithelial tumors of soft tissue: a clinicopathologic and immunohistochemical study of 101 cases with evaluation of prognostic parameters. Am J Surg Pathol. 2003;27:1183-96.

31. Gleason BC, Fletcher CD. Myoepithelial carcinoma of soft tissue in children: an aggressive neoplasm analyzed in a series of 29 cases. Am J Surg Pathol. 2007;31:1813-24. 
32. Hornick JL, Dal Cin P, Fletcher CD. Loss of INI1 expression is characteristic of both conventional and proximal-type epithelioid sarcoma. Am J Surg Pathol. 2009;33:542-50.

33. Stuart LN, Tipton RG, DeWall MR, Parker DC, Stelton CD, Morrison AO, et al. Primary cutaneous perivascular epithelioid cell tumor (PEComa): five new cases and review of the literature. J Cutan Pathol. 2017;44:713-21.

34. Charli-Joseph Y, Saggini A, Vemula S, Weier J, Mirza S, LeBoit PE, et al. Primary cutaneous perivascular epithelioid cell tumor: a clinicopathological and molecular reappraisal. J Am Acad Dermatol. 2014;71:1127-36.

35. Shon W, Billings SD. Cutaneous malignant vascular neoplasms. Clin Lab Med. 2017;37:633-46.

36. Ko JS, Billings SD. Diagnostically challenging epithelioid vascular tumors. Surg Pathol Clin. 2015;8:331-51.

37. Sangueza OP, Walsh SN, Sheehan DJ, Orland AF, Llombart B, Requena L, et al. Cutaneous epithelioid angiomatous nodule: a case series and proposed classification. Am J Dermatopathol. 2008;30:16-20.

38. Quante M, Patel NK, Hill S, Merchant W, Courtauld E, Newman $\mathrm{P}$, et al. Epithelioid hemangioendothelioma presenting in the skin: a clinicopathologic study of eight cases. Am J Dermatopathol. 1998;20:541-6.

39. Tanas MR, Sboner A, Oliveira AM, Erickson-Johnson MR, Hespelt J, Hanwright PJ, et al. Identification of a disease-defining gene fusion in epithelioid hemangioendothelioma. Sci Transl Med. 2011;3:98ra82.

40. Errani C, Zhang L, Sung YS, Hajdu M, Singer S, Maki RG, et al. A novel WWTR1-CAMTA1 gene fusion is a consistent abnormality in epithelioid hemangioendothelioma of different anatomic sites. Genes Chromosomes Cancer. 2011;50:644-53.

41. Antonescu CR, Le Loarer F, Mosquera JM, Sboner A, Zhang L, Chen CL, et al. Novel YAP1-TFE3 fusion defines a distinct subset of epithelioid hemangioendothelioma. Genes Chromosomes Cancer. 2013;52:775-84.

42. Doyle LA, Fletcher CD, Hornick JL. Nuclear expression of CAMTA1 distinguishes epithelioid hemangioendothelioma from histologic mimics. Am J Surg Pathol. 2016;40:94-102.

43. Deyrup AT, Tighiouart M, Montag AG, Weiss SW. Epithelioid hemangioendothelioma of soft tissue: a proposal for risk stratification based on 49 cases. Am J Surg Pathol. 2008;32:924-7.

44. Hornick JL, Fletcher CD. Pseudomyogenic hemangioendothelioma: a distinctive, often multicentric tumor with indolent behavior. Am J Surg Pathol. 2011;35:190-201.

45. Billings SD, Folpe AL, Weiss SW. Epithelioid sarcoma-like hemangioendothelioma. Am J Surg Pathol. 2003;27:48-57.

46. Walther C, Tayebwa J, Lilljebjorn H, Magnusson L, Nilsson J, von Steyern FV, et al. A novel SERPINE1-FOSB fusion gene results in transcriptional up-regulation of FOSB in pseudomyogenic haemangioendothelioma. J Pathol. 2014;232:534-40.

47. Sugita S, Hirano H, Kikuchi N, Kubo T, Asanuma H, Aoyama T, et al. Diagnostic utility of FOSB immunohistochemistry in pseudomyogenic hemangioendothelioma and its histological mimics. Diagn Pathol. 2016;11:75.

48. Hung YP, Fletcher CD, Hornick JL. FOSB is a useful diagnostic marker for pseudomyogenic hemangioendothelioma. Am J Surg Pathol. 2017;41:596-606.

49. Deyrup AT, McKenney JK, Tighiouart M, Folpe AL, Weiss SW. Sporadic cutaneous angiosarcomas: a proposal for risk stratification based on 69 cases. Am J Surg Pathol. 2008;32:72-7.

50. Fletcher CD, Beham A, Bekir S, Clarke AM, Marley NJ. Epithelioid angiosarcoma of deep soft tissue: a distinctive tumor readily mistaken for an epithelial neoplasm. Am J Surg Pathol. 1991;15:915-24.

51. Chase DR, Enzinger FM. Epithelioid sarcoma. Diagnosis, prognostic indicators, and treatment. Am J Surg Pathol. 1985;9: 241-63.

52. Spillane AJ, Meirion Thomas J, Fisher C. Epithelioid sarcoma: the clinicopathological complexities of this rare soft tissue sarcoma. Ann Surgical Oncol. 2000;7:218-25.

53. Guillou L, Wadden C, Coindre JM, Krausz T, Fletcher CD. "Proximal-type" epithelioid sarcoma, a distinctive aggressive neoplasm showing rhabdoid features. Clinicopathologic, immunohistochemical, and ultrastructural study of a series. Am J Surg Pathol. 1997;21:130-46.

54. Miettinen M, Fanburg-Smith JC, Virolainen M, Shmookler BM, Fetsch JF. Epithelioid sarcoma: an immunohistochemical analysis of 112 classical and variant cases and a discussion of the differential diagnosis. Hum Pathol. 1999;30:934-42.

55. Humble SD, Prieto VG, Horenstein MG. Cytokeratin 7 and 20 expression in epithelioid sarcoma. J Cutan Pathol. 2003;30:242-6.

56. Quezado MM, Middleton LP, Bryant B, Lane K, Weiss SW, Merino MJ. Allelic loss on chromosome 22q in epithelioid sarcomas. Hum Pathol. 1998;29:604-8.

57. Sonobe H, Ohtsuki Y, Sugimoto T, Shimizu K. Involvement of $8 \mathrm{q}, 22 \mathrm{q}$, and monosomy 21 in an epithelioid sarcoma. Cancer Genet Cytogenet. 1997;96:178-80.

58. Modena P, Lualdi E, Facchinetti F, Galli L, Teixeira MR, Pilotti $\mathrm{S}$, et al. SMARCB1/INI1 tumor suppressor gene is frequently inactivated in epithelioid sarcomas. Cancer Res. 2005;65:4012-9.

59. Hantschke M, Mentzel T, Rutten A, Palmedo G, Calonje E, Lazar AJ, et al. Cutaneous clear cell sarcoma: a clinicopathologic, immunohistochemical, and molecular analysis of 12 cases emphasizing its distinction from dermal melanoma. Am J Surg Pathol. 2010;34:216-22.

60. Falconieri G, Bacchi CE, Luzar B. Cutaneous clear cell sarcoma: report of three cases of a potentially underestimated mimicker of spindle cell melanoma. Am J Dermatopathol. 2012; $34: 619-25$

61. Kiuru M, Hameed M, Busam KJ. Compound clear cell sarcoma misdiagnosed as a Spitz nevus. J Cutan Pathol. 2013;40:950-4.

62. Yang L, Chen Y, Cui T, Knösel T, Zhang Q, Geier C, et al. Identification of biomarkers to distinguish clear cell sarcoma from malignant melanoma. Hum Pathol. 2012;43:1463-70. 